\title{
Impacto da reconstrução mamária na qualidade de vida de mulheres mastectomizadas
}

\author{
Impact of breast reconstruction on the quality of life of mastectomized women \\ Impacto de la reconstrucción mamaria en la calidad de vida de las mujeres \\ mastectomizadas
}

Eliane Patrícia Lemos de Almeida Leite ${ }^{1 *}$, Josefa Renata da Silva ${ }^{1}$, Suzana Elizabeth Buchegger Moura $^{1}$, Carina Batista de Paiva ${ }^{1}$, Themístoclys Thesko Correia Ferreira ${ }^{1}$.

\section{RESUMO}

Objetivo: Revisar as repercussões da reconstrução mamária na qualidade de vida das mulheres que foram submetidas à mastectomia. Metodologia: Estudo de revisão de literatura do tipo narrativa, realizada entre os meses de fevereiro a junho de 2019 , foi incluído estudos de coorte que avaliaram a qualidade de vida em mulheres que foram submetidas à reconstrução mamária através dos instrumentos: EORTC QLQ-C30 e BR23 e WHOQOL-100. Resultados: De acordo com os resultados apresentados nessa revisão, sobre as repercussões da reconstrução mamária na qualidade de vida de mulheres mastectomizadas, foram considerados resultados positivos para a imagem corporal, função sexual, prazer sexual, funcionamento psicológico e perspectivas Conclusão: A cirurgia reconstrutiva de mama proporcionou benefícios as pacientes que realizaram reconstrução mamária, mas, não há evidências suficientes para afirmar que melhora a qualidade de vida, devido a pouca quantidade de amostras e a escassez de estudos com metodologia de ensaio clínico randomizado.

Palavras-chave: Mamoplastia, Mastectomia, Qualidade de Vida.

\begin{abstract}
Objetive: To review the repercussions of breast reconstruction on the quality of life of women who underwent mastectomy. Methodology: A narrative literature review study conducted from February to June 2019 included cohort studies that assessed the quality of life in women who underwent breast reconstruction using the instruments: EORTC QLQ-C30 and BR23 and WHOQOL-100. Results: According to the results presented in this review, on the repercussions of breast reconstruction on the quality of life of mastectomized women, positive results for body image, sexual function, sexual pleasure, psychological functioning and perspectives were considered. Conclusion: Breast augmentation has provided benefits to patients who underwent breast reconstruction, but there is insufficient evidence to state that it improves quality of life due to the small sample size and the scarcity of randomized clinical trial studies.
\end{abstract}

Key words: Mammoplasty, Mastectomy, Quality of Life.

\section{RESUMEN}

Objetivo: Revisar las repercusiones de la reconstrucción mamaria en la calidad de vida de las mujeres que se sometieron a una mastectomía. Metodología: Un estudio de revisión de literatura narrativa realizado de febrero a junio de 2019 incluyó estudios de cohortes que evaluaron la calidad de vida en mujeres que se

${ }^{1}$ Centro Universitário Brasileiro (UNIBRA). Recife - PE. *E-mail: patyccbleite@hotmail.com 
sometieron a reconstrucción mamaria utilizando los instrumentos: EORTC QLQ-C30 y BR23 y WHOQOL100. Resultados: Según los resultados presentados en esta revisión, sobre las repercusiones de la reconstrucción mamaria en la calidad de vida de las mujeres mastectomizadas, se consideraron resultados positivos para la imagen corporal, la función sexual, el placer sexual, el funcionamiento psicológico y las perspectivas. Conclusión: El aumento de senos ha proporcionado beneficios a los pacientes que se sometieron a una reconstrucción de senos, pero no hay pruebas suficientes para afirmar que mejore la calidad de vida debido al pequeño tamaño de la muestra y la escasez de estudios que utilizan una metodología de ensayo controlado aleatorio.

Palabras clave: Mamoplastia, Mastectomía, Calidad de Vida.

\section{INTRODUÇÃO}

O câncer de mama é a neoplasia mais comum no mundo e a principal causa de mortalidade entre as mulheres, por isso é considerada um problema de saúde pública (INCA, 2019). No Brasil o Ministério da Saúde recomenda que seja realizado o exame mamográfico bienal em mulheres entre 50 e 69 anos, com a finalidade de rastrear a doença em seus estágios iniciais, pois quando diagnosticada e tratada precocemente pode promover um bom prognóstico (BARROS AES, et al., 2018; INCA, 2019).

A Organização Mundial de Saúde (2017), estima que no ano de 2030 ocorram 27 milhões de novos casos e 17 milhões de óbitos mundialmente. No Brasil, a taxa de mortalidade do câncer de mama mantém-se elevada e sua incidência para o biênio 2018-2019 é de 59.700 em mulheres com faixa etária entre 40 a 69 anos, representando cerca de $25 \%$ (vinte e cinco por cento) da neoplasia nessa população (BARROS AES, et al., 2018; INCA, 2019).

Dentre os fatores de risco para esta neoplasia maligna tem-se a obesidade, o sedentarismo, etilismo, exposição frequente a radiações ionizantes, menarca precoce, menopausa após os 55 anos, mulheres nulíparas, primeira gravidez após os 30 anos, uso de contraceptivos hormonais, reposição hormonal pósmenopausa, histórico familiar positivo, mutações nos genes BRCA 1 e BRCA 2, sendo a idade acima de 50 anos um dos fatores mais importante (INCA, 2019).

A neoplasia de mama traz preocupações às mulheres, sobretudo pelos impactos sociais e psicológicos que afetam a sua saúde, tais como: sofrer preconceitos; medo da recorrência ou morte; dor; alterações físicas; exclusão do meio social; comprometimento da sua autoimagem; disfunções sexuais; dificuldades na realização das atividades de vida diária e mudanças no estilo de vida (BARBOSA PA, et al., 2017).

O tratamento para o câncer de mama consiste em quimioterapia, radioterapia, hormonioterapia, como o tamoxifeno ou inibidores de aromatase e terapia alvo, como exemplo o trastuzumabe (INCA, 2018). O tratamento cirúrgico é dividido em cirurgias radicais e conservadoras, porém, tais abordagens proporcionam comorbidades que afetam a qualidade de vida dessas mulheres, como a dor e a restrição da mobilidade do membro homolateral (MARTINS TNO, et al., 2017)

Para as mulheres a mama representa sua feminilidade, sexualidade, maternidade e autoestima, por isso, os especialistas vêm se preocupando cada vez mais com a implantação de novas técnicas capazes de produzir uma mama idêntica em forma e aparência à mama natural (INOCENTI A, et al., 2016).

Nesse contexto, os avanços das técnicas cirúrgicas oncoplásticas proporcionam a reconstrução mamária, que pode ser imediata, tardia, com ou sem uso de expansor, implantes de silicone e enxertos com tecidos autólogos (retalho miocutâneo transverso do abdome, e retalho do músculo grande dorsal) com intuito de devolver à mulher a mama mutilada ou deformada (DIAS LV, et al., 2017; FREIRE MM, et al., 2017; BARROS AES, et al., 2018).

A reconstrução de mama tem o objetivo de melhorar a Qualidade de Vida $(\mathrm{QV})$ as pacientes, pois esse procedimento pode amenizar o impacto emocional e físico causado pela cirurgia. A mesma tem como escopo imediato a reparação da mutilação, recondicionando a forma e o volume da mama, conservando assim a 
autoimagem da mulher mastectomizada, impactando diretamente no seu contexto emocional, físico e social (INOCENTI A, et al., 2016).

Diante disto, essa revisão de literatura teve por objetivo revisar as repercussões da reconstrução mamária na qualidade de vida das mulheres que foram submetidas à cirurgia do tipo mastectomia.

\section{MÉTODOS}

O presente estudo trata-se de uma revisão bibliográfica do tipo narrativa em que realizou-se uma busca de publicações sobre o tema em questão, entre os meses de fevereiro a junho de 2019, através das bases de dados online: Medical Literature Analsis and Retrieval System (Medline) via Pubmed, Literatura Latino Americana e do Caribe da Ciência em Saúde (LILACS) via Centro Latino Americano e do Caribe de Informação em Ciências da Saúde (BIREME), Scientific Electronic Library Online (SCIELO). Foram utilizados artigos sem restrição linguística, com restrição temporal dos últimos 10 anos. Não foram utilizados artigos de outras fontes.

A estratégia de busca baseou-se nos descritores cadastrados nos Descritores de Ciência da Saúde (DECS): mamoplastia, reconstrução de mama, European Organization for Research and Treatment of Cancer Quality of Life Questionnaire (EORTC QLQ), World Health Organization Quality of life (WHOQOL) e seus respectivos corretores em inglês cadastrados no Medical Subject Headings (MESH): mammoplasty, breast reconstruction, EORTC QLQ, WHOQOL, combinados entre si pelo operador booleano AND e OR, conforme descritos no (Quadro 1).

Quadro 1 - Busca de dados através da estratégia da combinação dos descritores.

\begin{tabular}{|c|c|}
\hline BASE DE DADOS & ESTRATÉGIA DE BUSCA \\
\hline Medline/PUBMED & 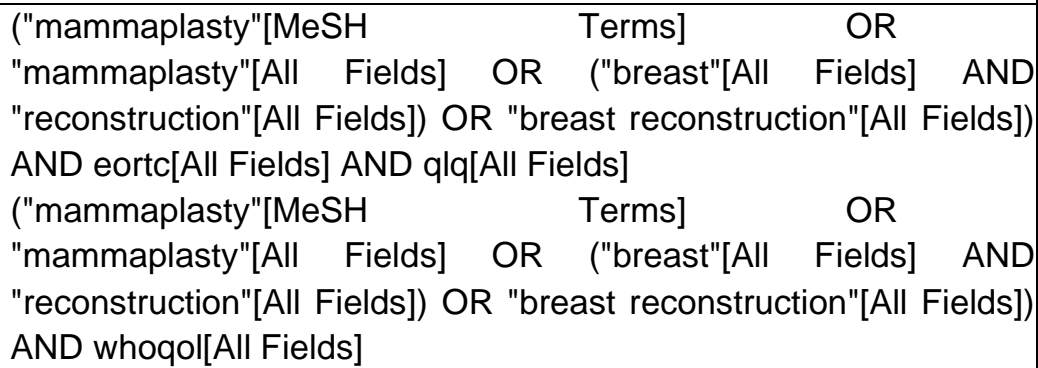 \\
\hline LILACS/BIREME & $\begin{array}{l}\text { tw:(reconstrução de mama AND eortc ) AND (instance:"regional") } \\
\text { tw:(reconstrução de mama AND whoqol) AND } \\
\text { (instance:"regional") }\end{array}$ \\
\hline SCIELO & $\begin{array}{l}\text { Reconstrução de mama and eortc } \\
\text { Reconstrução de mama AND whoqol }\end{array}$ \\
\hline
\end{tabular}

Fonte: Leite EPLA, et al. 2019.

Os critérios de inclusão foram os estudos que avaliaram a qualidade de vida em mulheres que foram submetidas à reconstrução mamária, sendo elas de qualquer faixa etária. Também foram motivos de inclusão estudos quantitativos que utilizaram questionários validados para a avaliação de qualidade de vida.

Sendo elegíveis os estudos que avaliaram qualidade de vida utilizando os seguintes instrumentos: questionário de qualidade de vida da European Organization for Research and Treatment of Cancer Breast Cancer-Specific Quality of Life (EORTC QLQ-C30 e EORTC QLQ-BR23), pois é um instrumento que avalia especificamente a qualidade de vida no câncer, validado mundialmente e o questionário World Health Organization Quality of life (WHOQOL-100) proposto pela organização mundial de saúde, validado para a população brasileira, com critérios relevantes para avaliar a qualidade de vida em pacientes com câncer de mama. Também como critério de inclusão estudos que consistissem em ensaios clínicos randomizados ou quase randomizados, no entanto, na ausência desses estudos foram utilizados estudos de coorte. 
Foram excluídos artigos que utilizaram outros métodos de tratamento e que fugiam do tema, duplicatas, sendo também motivo de exclusão estudos que não se enquadravam em ensaios clínicos randomizados, quase randomizados ou de coorte. A seleção dos artigos foi realizada através da leitura de títulos e resumos, logo após, realizou-se a leitura dos artigos na íntegra. Os artigos selecionados foram na língua inglesa e portuguesa, estudos de coorte prospectivo e retrospectivo. A seleção dos estudos identificados pela busca foi realizada segundo os critérios de elegibilidade proposto pelo fluxograma desenvolvido pelo the PRISMA statement (Figura 1).

Figura 1 - Busca a seleção dos artigos para a revisão de literatura de acordo com o PRISMA.
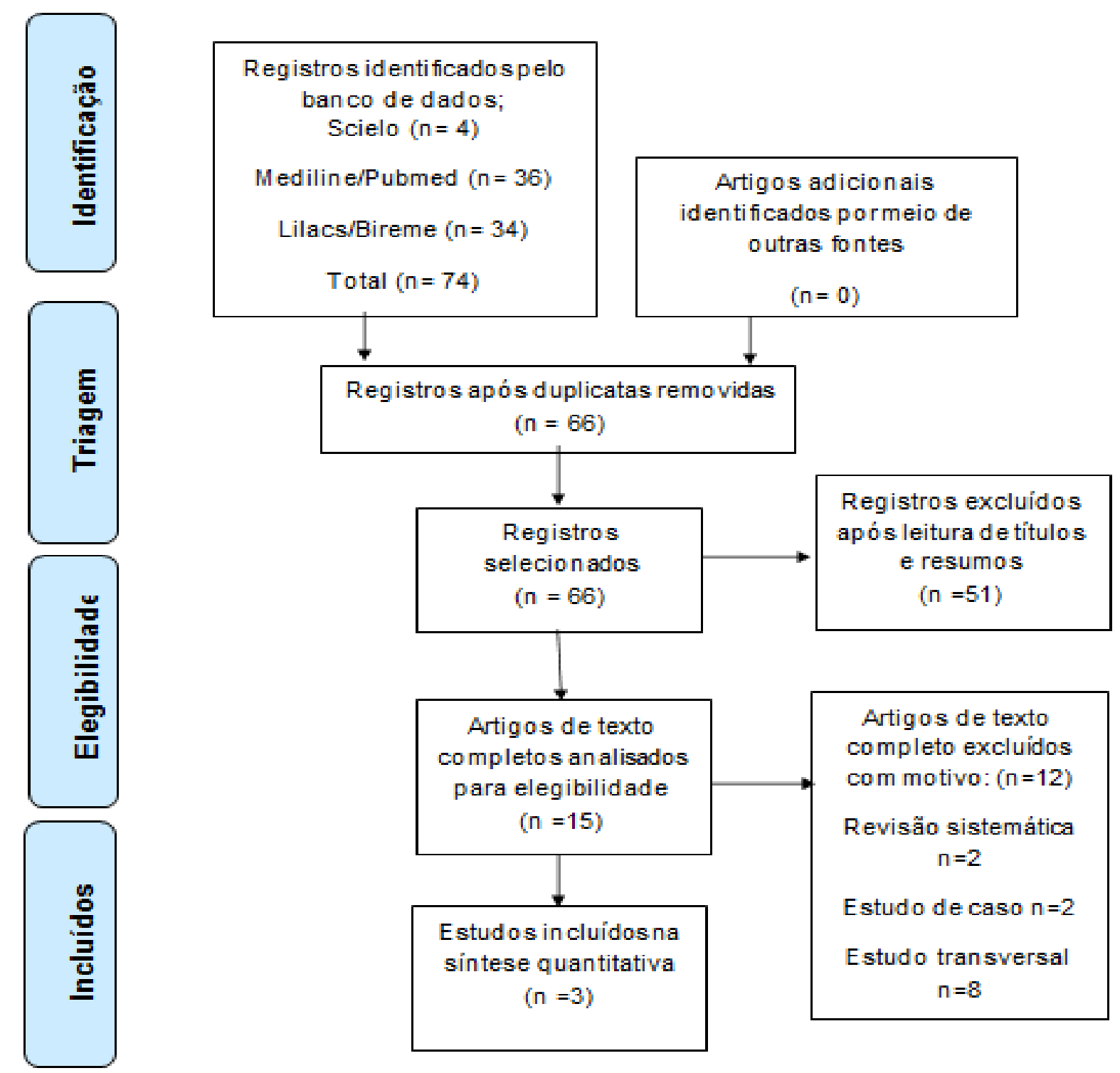

Fonte: Leite EPLA, et al. 2019.

\section{RESULTADOS e DISCUSSÃO}

Esta pesquisa incluiu o total de 3 artigos que utilizaram os questionários validados para avaliar a qualidade de vida de mulheres que realizaram reconstrução mamária, sendo eles: o estudo de Min SY, et al., (2010), Oliveira RR, et al., (2010), e Spatuzzi R, et al., (2016), conforme descrito no (Quadro 2) que apresenta as especificações dos artigos segundo autor/ano da publicação, tipo de estudo, amostra, objetivo, instrumento de avaliação e resultados principais. 
Quadro 2 - Apresentação da síntese dos artigos incluídos na revisão.

\begin{tabular}{|c|c|c|c|c|c|}
\hline Autor/ano & Tipo de Estudo & Amostra & Objetivo & Método de Avaliação & Resultados \\
\hline $\begin{array}{l}\text { Min SY, } \\
\text { et al. (2010) }\end{array}$ & $\begin{array}{l}\text { Coorte } \\
\text { retrospectivo }\end{array}$ & $\begin{array}{l}\mathrm{N}=2.566 \\
\text { Mulheres coreanas } \\
<40 \text { anos e > } 40 \text { anos } \\
\text { Grupo: RGD } \\
\text { Reconstrução mamária } \\
\text { imediata com RGD }(\mathrm{n}=52) \\
\text { Grupo: BCS } \\
\text { Cirurgia conservadora de } \\
\text { mama ( } \mathrm{n}=104)\end{array}$ & $\begin{array}{l}\text { Comparar a qualidade de } \\
\text { vida de pacientes } \\
\text { submetidas à cirurgia } \\
\text { conservadora de mama em } \\
\text { relação aquelas que } \\
\text { fizeram reconstrução } \\
\text { mamária imediata com } \\
\text { RGD }\end{array}$ & $\begin{array}{l}\text { EORTC } \\
\text { QLQ-BR23 } \\
\text { SDS }\end{array}$ & $\begin{array}{l}\text { O grupo RGD correlacionou-se } \\
\text { positivamente com a função e o } \\
\text { prazer sexual, o grupo BCS } \\
\text { correlacionou-se positivamente } \\
\text { com a imagem corporal e } \\
\text { perspectivas futuras e tiveram } \\
\text { diferenças estatisticamente } \\
\text { significativas de p=0,001 e } \\
\text { p<0,001 respectivamente. Não } \\
\text { apresentaram alterações na } \\
\text { escala de depressão, escore }<49 .\end{array}$ \\
\hline $\begin{array}{l}\text { Oliveira RR, et } \\
\text { al. (2010) }\end{array}$ & $\begin{array}{l}\text { Coorte } \\
\text { prospectivo }\end{array}$ & $\begin{array}{l}\mathrm{N}=76 \\
\text { Mulheres Brasileiras } \\
\text { Grupo:M+RI, aprox.43 anos. } \\
\text { Mastectomia associada à } \\
\text { Reconstrução imediata da } \\
\text { mama ( } \mathrm{n}=41) \\
\text { Grupo: M aprox. } 57 \text { anos, } \\
\text { Mastectomia } \\
\text { reconstrução }(\mathrm{n}=35)\end{array}$ & $\begin{array}{l}\text { Avaliar prospectivamente } \\
\text { os efeitos da reconstrução } \\
\text { mamária imediata sobre a } \\
\text { qualidade de vida de } \\
\text { mulheres mastectomizadas }\end{array}$ & WHOQOL-100 & $\begin{array}{l}\text { Houve melhores pontuações nas } \\
\text { avaliações de qualidade de vida } \\
\text { no grupo } M+R I \text { em relação aos } \\
\text { domínios: psicológico e aspectos } \\
\text { espirituais, } p<0,001 \text {. Não ocorreu } \\
\text { impacto da reconstrução mamária } \\
\text { nos domínios físicos, sociais e de } \\
\text { meio ambiente. }\end{array}$ \\
\hline
\end{tabular}

REAS/EJCH | Vol.Sup.35 | e1502 | DOI: https://doi.org/10.25248/reas.e1502.2019 Página 5 de 8 


\begin{tabular}{|c|c|c|c|c|c|}
\hline $\begin{array}{l}\text { Spatuzzi } \\
\text { et al. (2016) }\end{array}$ & $\begin{array}{l}\text { Coorte } \\
\text { retrospectivo }\end{array}$ & $\begin{array}{l}\mathrm{N}=157 \\
\text { Idade 35-75 anos } \\
\text { Mulheres Italianas } \\
\text { Grupo: BCS } \\
\text { Cirurgia conservadora de } \\
\text { mama ( } \mathrm{n}=72 \text { ) } \\
\text { Grupo: M } \\
\text { Mastectomia } \\
\text { reconstrução } \\
\text { ( } \mathrm{n}=44 \text { ) } \\
\text { Grupo: } \mathrm{R} \\
\text { Reconstrução mamária após } \\
\text { mastectomia ( } \mathrm{n}=41 \text { ) }\end{array}$ & $\begin{array}{l}\text { Comparar a qualidade de } \\
\text { vida, imagem corporal e } \\
\text { suporte social percebido } \\
\text { em mulheres com cirurgia } \\
\text { de câncer de mama. }\end{array}$ & $\begin{array}{l}\text { EORTC QLQ-C30 } \\
\text { EORTC QLQ- BR23 } \\
\text { BIS } \\
\text { PSS }\end{array}$ & $\begin{array}{l}\text { Houve diferença significativa entre } \\
\text { os três grupos no domínio } \\
\text { funcional do EORTC QLQ-C30 e } \\
\text { BR23, sendo a maior pontuação } \\
\text { no grupo R. } \\
\text { Em relação ao suporte social, o } \\
\text { grupo BCS mostrou mais apoio } \\
\text { que o grupo M, no grupo R foi } \\
\text { encontrado correlação negativa } \\
\text { entre um PSS positivo e alteração } \\
\text { na imagem corporal. Houve } \\
\text { diferença de escores da BIS entre } \\
\text { M e R P>0,05 e PSS apresentou } \\
\text { p<0,001. } \\
\text { Não houve diferença da qualidade } \\
\text { de vida com relação a imagem } \\
\text { corporal e o suporte social } \\
\text { percebido. }\end{array}$ \\
\hline
\end{tabular}

Legenda: RGD: retalho do grande dorsal, BCS: breast conserving surgery, EORTC QLQ-BR23: European Organization for Research and Treatment of Cancer Breast Cancer-Specific Quality of Life, SDS Zung's: self-rating depression scale, M: mastectomia, RI: reconstrução imediata, Aprox.: Aproximadamente, WHOQOL100: World Health Organization Quality of life, R: reconstrução, EORTC QLQ-C30: European Organization for Research and Treatment of Cancer Breast CancerSpecific Quality of Life, BIS: body image scaling, PSS: perceived social support scale.

Fonte: Leite EPLA, et al. 2019.

REAS/EJCH | Vol.Sup.35 | e1502 | DOI: https://doi.org/10.25248/reas.e1502.2019 Página 6 de 8 
Diante da pesquisa realizada pelas bases de dados obteve-se um total de 74 artigos. Dos 74 artigos, foram excluídos 8 estudos por serem duplicatas, 51 após a leitura de títulos e resumos, por não se adequarem aos critérios de inclusão: estudos que avaliassem a qualidade de vida em mulheres que foram submetidas à reconstrução mamária, por meio dos questionários Scale of the European Organization for Cancer Research and treatment, titled "Quality of Life Questionnaire version 3.0 (Escala da Organização Europeia para Pesquisa e Tratamento do Câncer, intitulado "Questionário de Qualidade de Vida versão 3.0) (EORTC QLQ-C30), Scale of the European Organization for Breast Cancer Research and treatment (Escala da Organização Europeia para Pesquisa e Tratamento do câncer de mama) (EORTC QLQ-BR23) e The World Health Organization instrument to evaluate quality of life (Instrumento de avaliação de Qualidade de Vida da Organização Mundial de Saúde) (WHOQOL-100), 12 estudos foram excluídos após a leitura do texto completo, por motivo de não serem estudos randomizados, quase randomizados ou estudos de coorte.

É presente na literatura cientifica estudos que verificam o aumento da insatisfação de mulheres submetidas às cirurgias de mastectomias, como forma de tratamento para o câncer de mama, pois há nessas abordagens cirúrgicas perceptíveis mudanças na imagem corporal, fator este que pode ser associado com grandes prejuízos na percepção de Qualidade de Vida (QV) por essas pacientes. Sendo a reconstrução mamária uma abordagem que pode ser associada a uma melhor percepção de QV (PRATES ACL, et al. 2017).

Foi observado no estudo de Min SY, et al., (2010), que o grupo que fez reconstrução de mama imediata com o retalho do grande dorsal apresentaram um escore significativamente baixo de 64.9 para imagem corporal e 49.0 para perspectivas futuras, do que o grupo que fez cirurgia conservadora que obteve melhores resultados nesses domínios, 73.0 e 58.6 respectivamente. Sendo observado que as mulheres que realizaram reconstrução mamária, a retirada da mama teve grande impacto. Em contrapartida, no estudo de Spatuzzi R, et al (2016), as mulheres que foram submetidas a reconstrução de mama não tiveram dificuldades com sua imagem corporal.

Ainda se tratando do estudo de Spatuzzi R, et al., (2016), que avaliaram o domínio sintoma através dos questionários EORTC QLQ-C30 e QLQ-BR23, verificaram a diferença entre os grupos apenas para a escala de dispneia, mas, em relação a imagem corporal, o grupo de cirurgia conservadora foi comparado ao grupo de mastectomia e demostraram superioridade na percepção da imagem corporal.

Da mesma forma, Min SY, et al., (2010), verificaram que os escores de imagem corporal e perspectivas futuras foram significativamente menores para as mulheres submetidas à reconstrução do que as submetidas à cirurgia conservadora, porém, não utilizaram todo o EORTC QLQ-BR 23 e não avaliaram os escores funcionais e emocionais, mas, evidenciaram que a mastectomia seguida de reconstrução imediata com o retalho do grande dorsal não necessariamente fornece uma melhor qualidade de vida que a cirurgia conservadora.

Já no estudo de Spatuzzi R, et al., (2016), enfatizaram a importância do apoio social da família e outros, para as mulheres submetidas a cirurgia de mama, pois neste estudo verificaram que as mulheres que decidiram fazer a reconstrução mamária tiveram um nível de satisfação positivo por meio do suporte social em relação a sua imagem corporal, mas, devido a amostra ser considerada pequena não levaram em consideração que a imagem corporal pode influenciar fortemente a qualidade de vida.

Em conformidade com Min SY, et al., (2010) e Spatuzzi R, et al., (2016), finalizaram seu estudo assegurando não ser possível analisar o impacto da reconstrução mamária comparada com a imagem corporal e o suporte social, pois a cirurgia reconstrutiva de mama após a mastectomia geralmente é entendida que melhora a percepção da imagem corporal comparada com a mastectomia sozinha, mas, em outros aspectos da qualidade de vida e bem-estar das pacientes não parece diferir.

Da mesma maneira, Oliveira RR, et al., (2010), avaliaram a qualidade de vida por meio do questionário WHOQOQ-100, proposto pela Organização Mundial de Saúde, validado, em que observaram que as mulheres que realizaram a reconstrução de mama imediata alcançaram melhores pontuações nas avaliações da qualidade de vida nos domínios: psicológico e aspectos espirituais. Ao mesmo tempo, a reconstrução mamária imediata na vida dessas mulheres não trouxe impacto nos domínios físicos, sociais e de meio 
ambiente. Ainda se tratando do estudo de Oliveira RR, et al., (2010), demostraram que a cirurgia conservadora provavelmente tenha efeito benéfico sobre a aceitação psicológica do tratamento, no entanto, essas diferenças são possíveis de perceber quando ocorre uma análise de resultado de maneira distinta, pois o câncer, por si só, produz efeito psicológico negativo. Por isso, mesmo as mulheres sendo tratadas com a reconstrução de mama, podem apresentar perda do domínio psicológico, mas por serem beneficiadas pela reconstrução mamária imediata apresentam melhores resultados nas avaliações de qualidade de vida.

Min SY, et al., (2010), ressaltam em seu estudo que não há evidências de que as mulheres coreanas sejam diferentes biologicamente das mulheres em outros países, tornando assim, um pouco diferente a percepção da imagem corporal das culturas coreanas relacionadas as mulheres dos países ocidentais.

\section{CONSIDERAÇÕES FINAIS}

De acordo com os resultados apresentados nessa revisão, foram considerados resultados positivos para a imagem corporal, função sexual, prazer sexual, funcionamento psicológico e perspectivas. Os artigos selecionados mostraram que a cirurgia reconstrutiva de mama, proporcionou benefícios as pacientes que realizaram reconstrução mamária, mas, não há evidências suficientes para afirmar que melhora a qualidade de vida, devido à pouca quantidade de amostras e a escassez de estudos com metodologia de ensaio clínico randomizado. Seria necessário que os estudos fossem homogêneos na estratificação das idades das mulheres, para entender melhor às repercussões da reconstrução mamária na qualidade de vida das mulheres submetidas à reconstrução mamária.

\section{REFERÊNCIAS}

1. BARBOSA PA, et al. Qualidade de vida em mulheres com câncer de mama pós-intervenção cirúrgica em uma cidade da zona da mata de Minas Gerais, Brasil. Revista Brasileira de Saúde Materno Infantil, 2017; 17(2): 401-416.

2. BARROS AES. Feelings experienced by women whem receiving the diagnosis of breast cancer. Revista de Enfermagem Online, 2018; 12(1): 102-111.

3. DIAS LV, et al. Mulher mastectomizada por câncer de mama: vivência das atividades cotidianas. Revista Cuidado é Fundamental Online, 2017; 9(4): 1074-1080.

4. FREIRE, M. M. et al. Breast cancer and its treatments: repercissions in sexuality lived by women. Revista Enfermagem UFPE Online, 2017; 11(11): 4511-4514.

5. INOCENTI A, et al. Repercussão dos efeitos da cirurgia reconstrutora na vida de mulheres com neoplasias de mama. Revista Texto Contexto Enfermagem, 2016; 25 (2): 1-9.

6. INSTITUTO NACIONAL DO CÂNCER. INCA. Brasil, 2018. Estimativa: incidência de câncer no Brasil. Disponível em: https://www.inca.gov.br/publicacoes/livros/estimativa-2018-incidencia-de-cancer-no-brasil. Acesso em: 16 abr. 2019.

7. INSTITUTO NACIONAL DO CÂNCER. INCA. Brasil, 2018. Tratamento para o câncer de mama. Disponível em: https://www.inca.gov.br/controle-do-cancer-de-mama/acoes-de-controle/tratamento. Acesso em: 28 mar. 2019.

8. INSTITUTO NACIONAL DO CÂNCER. INCA. Brasil, 2018. Novos construtores de autoestima. Disponível em: https://www.inca.gov.br/sites/ufu.sti.inca.local/files//media/document//rrc-27-assistencia-novos-construtores-deautoestima.pdf. Acesso em: 28 abr. 2019.

9. INSTITUTO NACIONAL DO CÂNCER. INCA. Brasil, 2019. Fatores de risco para o câncer de mama. Disponível em: https://www.inca.gov.br/controle-do-cancer-de-mama/fatores-de-risco. Acesso em: 18 abr. 2019.

10. MARTINS TNO, et al. Reconstrução mamária imediata versus não reconstrução pós-mastectomia: estudo sobre qualidade de vida, dor e funcionalidade. Fisioterapia Pesquisa, 2017; 24(4): 412-419.

11. MIN SY, et al. Oncological safety and quality of life associated with mastectomy and immediate breast reconstruction with a latissimus dorsi myocutaneous flap. The Breast Journal, 2010; 16(4): 356-361.

12. OLIVEIRA RR, et al. Efeitos da reconstrução mamária imediata sobre a qualidade de vida de mulheres mastectomizadas. Revista Brasileira de Ginecologia e Obstetrícia, 2010; 32(12): 602-608.

13. PRATES ACL, et al. Influence of Body Image in Women Undergoing Treatment for Breast Cancer. Revista Brasileira de Ginecologia e Obstetrícia. 2017; 39(4):175-83.

14. SPATUZZI R, et al. Evaluation of Social Support, Quality of Life, and Body Image in Women with Breast Cancer. Breast Care, 2016; 11(1): 28-32. 